04,09

\title{
Правило Урбаха и оценка ширины запрещенной зоны в молибдатах
}

\author{
(С) Ф.Д. Федюнин ${ }^{1}$, Д.А. Спасский ${ }^{2}$ \\ ${ }^{1}$ Московский государственный университет им. М.В. Ломоносова, \\ Москва, Россия \\ ${ }^{2}$ Научно-исследовательский институт ядерной фризики им. Д.В. Скобельцына \\ Московского государственного университета им. М.В. Ломоносова, \\ Москва, Россия \\ E-mail: daspassky@gmail.com
}

Поступила в Редакцию 6 марта 2020 г.

В окончательной редакции 6 марта 2020 г.

Принята к публикации 1 апреля 2020 г.

В настоящей работе проведено исследование температурной зависимости края фундаментального поглощения молибдатов $\mathrm{CaMoO}_{4}, \mathrm{SrMoO}_{4}, \mathrm{PbMoO}_{4}, \mathrm{~Pb}_{2} \mathrm{MoO}_{5}$ и $\mathrm{MgMoO}_{4}$. Полученные зависимости были аппроксимированы с использованием формулы Урбаха. Показано, что параметр $E_{0}$, полученный в результате аппроксимации, может быть использован для оценки ширины запрещенной зоны молибдатов.

Ключевые слова: правило Урбаха, молибдаты, экситоны, ширина запрещенной зоны.

DOI: 10.21883/FTT.2020.08.49598.052

\section{1. Введение}

Кристаллы молибдатов являются перспективными материалами для применения в качестве криогенных сцинтилляционных детекторов [1-5]. Такие детекторы используются для регистрации редких событий, например, двойного бета распада $(2 v \beta \beta)$, двойного безнейтринного бета распада $(0 v \beta \beta)$ и взаимодействия вещества с темной материей. Интерес к молибдатам обуславливается тем, что изотоп молибдена ${ }^{100}$ Мо является потенциальным источником двойного безнейтринного бета распада благодаря совокупности свойств, таких как высокая энергия процесса, большая изотопная распространенность ${ }^{100} \mathrm{Mo}$, малое предполагаемое время полураспада $[6,7]$. Таким образом, существенным достоинством кристаллов молибдатов является то, что они представляют собой как источник редкого события (например, $0 v \beta \beta)$, так и его детектор, что позволяет минимизировать энергетические потери и увеличить вероятность регистрации события.

В настоящее время целый ряд молибдатов рассматривается на роль сцинтиллятора для криогенных болометров $[8,9]$. Однако, изученные молибдаты наряду с достоинствами, имеют также свои недостатки. Например, молибдат кальция характеризуется наиболее высоким значением сцинтилляционного выхода среди молибдатов (до 10000 phot/MeV в криогенных условиях), однако наличие у катиона изотопа ${ }^{48} \mathrm{Ca}$, который является источником процесса $2 v \beta \beta$, создает неустранимый фон при регистрации процесса $0 v \beta \beta$. Проблема наличия радиоактивных изотопов характерна и для других катионов в молибдатах, например свинца, стронция, кадмия. Молибдаты с легкими катионами ( $\mathrm{Li}, \mathrm{Na}, \mathrm{Mg}$ ) лишены этого недостатка, однако молибдаты лития и магния характеризуются низкими значениями светового выхода, а монокристаллы молибдатов натрия и лития достаточно сложны в обработке [10-13]. Таким образом, проблема выбора наиболее подходящего кристалла до сих пор остается актуальной.

Экситоны играют важную роль в сцинтилляционном процессе молибдатов. Так, люминесценция молибдатов связана с излучательной рекомбинацией экситонов, автолокализованных на $\mathrm{MoO}_{4}^{2-}$ комплексах [14-17]. Как правило, сцинтилляционный выход молибдатов с собственной люминесценцией автолокализованных экситонов практически не уменьшается в результате захвата электронов и дырок на мелких ловушках, а также в результате их автолокализации [18-20]. Это является преимуществом перед традиционными сцинтилляторами c активаторной люминесценцией (например, NaI:Tl, $\mathrm{CsI}: \mathrm{Tl}, \mathrm{Y}_{3} \mathrm{Al}_{5} \mathrm{O}_{12}$ : Се, и т.д.), сцинтилляционный выход которых существенно уменьшается при понижении температуры [21-24].

Одним из проявлений существования экситонов в кристаллах является характерная форма края фундаментального поглощения (ФП). Экситон-фононное взаимодействие определяет экспоненциальную форму края ФП, а также смещение края при изменении температуры [25]. При этом температурная зависимость края ФП может быть описана правилом Урбаха [26-29]. Насколько нам известно, ранее исследования соответствия формы края ФП правилу Урбаха были проведены только для кристаллов молибдата свинца [30,31] и магния [15].

Целью работы являлось исследование температурной зависимости края фундаментального поглощения и ее аппроксимация согласно правилу Урбаха, а также анализ возможности использования результатов аппроксимации для оценки ширины запрещенной зоны молибдатов $\mathrm{CaMoO}_{4}, \mathrm{SrMoO}_{4}, \mathrm{PbMoO}_{4}, \mathrm{~Pb}_{2} \mathrm{MoO}_{5}$ и $\mathrm{MgMoO}_{4}$. 


\section{2. Техника эксперимента}

Спектры поглощения и отражения монокристаллических плоскопараллельных пластин молибдатов были измерены с использованием спектрофотометра Perkin-Elmer Lambda-950 в температурном диапазоне $77-500$ К. Образцы были помещены в оптический вакуумный криостат Cryotrade LN-120. Контроль и управление температурой осуществлялись с использованием температурного контроллера LakeShore-335.

Кристаллы были выращены методом Чохральского $\left(\mathrm{CaMoO}_{4}, \mathrm{SrMoO}_{4}, \mathrm{PbMoO}_{4}, \mathrm{MgMoO}_{4}\right)$, а также модифицированным методом Чохральского с низким температурным градиентом $\left(\mathrm{Pb}_{2} \mathrm{MoO}_{5}\right)$. Кристалл $\mathrm{CaMoO}_{4}$ был выращен в ФОМОС-Материалс, кристаллы $\mathrm{SrMoO}_{4}$ и $\mathrm{MgMoO}_{4}$ - в ИОФ РАН им. А.М. Прохорова, $\mathrm{Pb}_{2} \mathrm{MoO}_{5}$ - в ИНХ СО РАН им. А.В. Николаева, $\mathrm{PbMoO}_{4}$ - во ВНИИСИМС. Измерения были проведены для полированных плоскопараллельных пластин монокристаллов $\mathrm{CaMoO}_{4}$ толщиной $2 \mathrm{~mm}, \mathrm{SrMoO}_{4}$ толщиной $1.8 \mathrm{~mm}, \mathrm{PbMoO}_{4}$ толщиной $1.1 \mathrm{~mm}$, а также для плоскопараллельных сколов монокристаллов $\mathrm{MgMoO}_{4}$ толщиной $0.6 \mathrm{~mm}$ и $\mathrm{Pb}_{2} \mathrm{MoO}_{5}$ толщиной $0.4 \mathrm{~mm}$. Исследованные кристаллы молибдатов характеризуются разной кристаллической структурой. Кристаллы $\mathrm{CaMoO}_{4}$, $\mathrm{SrMoO}_{4}$ и $\mathrm{PbMoO}_{4}$ относятся к тетрагональному структурному типу шеелита; $\mathrm{Pb}_{2} \mathrm{MoO}_{5}$ относится к структурному типу ланаркита; $\mathrm{MgMoO}_{4}$ относится к структурному типу $\beta$ - $\mathrm{MgMoO}_{4}$ [32-34].

\section{3. Результаты}

Температурные зависимости спектров поглощения молибдатов представлены на рис. 1. Резкий рост коэффициента поглощения в спектрах соответствует области края фундаментального поглощения кристаллов. При повышении температуры край ФП смещается в низкоэнергетическую область. Предположительно край ФП в молибдатах связан с созданием экситонов, а температурная зависимость края определяется изменением величины экситон-фононного взаимодействия, так что с увеличением температуры полоса экситонного поглощения уширяется. При этом поведение низкоэнергетического края полосы поглощения экситонов описывается правилом Урбаха: [25-29]:

$$
\alpha(E)=\alpha_{0} \exp \left(-\sigma(T) \frac{E_{0}-E}{k T}\right),
$$

где $\sigma(T)$ - эмпирический коэффициент наклона, $\alpha_{0}-$ коэффициент поглощения при $E=E_{0}, k-$ константа Больцмана, $E$ - энергия падающего излучения, $T-$ температура образца.

В соответствии с правилом коэффициент поглощения вещества увеличивается экспоненциально с увеличением энергии фотона, т.е. логарифмы коэффициента поглощения, построенные как функции от энергии фотона,
Таблица 1. Параметры аппроксимации температурной зависимости края фундаментального поглощения молибдатов по правилу Урбаха

\begin{tabular}{c|c|c|c|c}
\hline Молибдаты & $E_{0}, \mathrm{eV}$ & $\sigma_{0}$ & $\alpha_{0}, \mathrm{~cm}^{-1}$ & $\hbar \omega, \mathrm{meV}$ \\
\hline $\mathrm{CaMoO}_{4}$ & $4.76 \pm 0.03$ & 0.54 & $(806 \pm 18) \cdot 10^{3}$ & 78 \\
$\mathrm{SrMoO}_{4}$ & $4.75 \pm 0.01$ & 0.50 & $(893 \pm 8) \cdot 10^{2}$ & 68 \\
$\mathrm{PbMoO}_{4}$ & $3.71 \pm 0.03$ & 0.53 & $(269 \pm 8) \cdot 10^{2}$ & 52 \\
$\mathrm{~Pb}_{2} \mathrm{MoO}_{5}$ & $4.25 \pm 0.05$ & 0.57 & $(327 \pm 15) \cdot 10^{4}$ & 59 \\
$\mathrm{MgMoO}_{4}$ & $4.97 \pm 0.03$ & 0.55 & $(162 \pm 5) \cdot 10^{4}$ & 90
\end{tabular}

могут быть аппроксимированы прямой. Аппроксимация производилась при помощи метода наименьших квадратов. Согласно [35], данная зависимость допускает линеаризацию путем логарифмирования уравнения, что позволяет применить метод наименьших квадратов. В результате был получен набор прямых, сходящихся в некоторой области (в идеальном случае - в точке). Для определения точки пересечения усреднялись координаты всех попарных пересечений прямых. Погрешность для $E_{0}$ и $\ln \left(\alpha_{0}\right)$ определялась как стандартное отклонение от среднего для точек пересечения. Погрешность для параметра $\alpha_{0}$ определялась как произведение $\alpha_{0}$ и относительной ошибки для величины $\ln \left(\alpha_{0}\right)$. Полученные в результате аппроксимации параметры $E_{0}$ и $\alpha_{0}$ для пяти молибдатов приведены в табл. 1. Отметим, что аппроксимация спектров поглощения $\mathrm{PbMoO}_{4}$ с использованием (1) возможна в относительно узком диапазоне значений коэффициента поглощения. Это связано с наличием в области прозрачности кристалла пика поглощения, связанного с дефектами структуры. Отметим также, что линии аппроксимации кривых, измеренных при $T=100 \mathrm{~K}$ для кристаллов $\mathrm{PbMoO}_{4}$ и $\mathrm{Pb}_{2} \mathrm{MoO}_{5}$, отклоняются от области схождения аппроксимирующих линий для других температур. Это может быть связано с искажением низкотемпературного края фундаментального поглощения полосами поглощения дефектов, которые расположены за краем фундаментального поглощения. Значения $E_{0}$, определенные по кривым поглощения при температурах $200-500 \mathrm{~K}$, составляют $3.75 \pm 0.02$ и $4.16 \pm 0.01 \mathrm{eV}$ для кристаллов $\mathrm{PbMoO}_{4}$ и $\mathrm{Pb}_{2} \mathrm{MoO}_{5}$ соответственно.

В некоторых моделях, которые характеризуются взаимодействием экситона с одним типом оптических фононов, температурная зависимость коэффициента наклона может быть представлена как

$$
\sigma(T)=\sigma_{0} \frac{2 k T}{\hbar \omega} \operatorname{th}\left(\frac{\hbar \omega}{2 k T}\right),
$$

где $\hbar \omega-$ энергия фононов, $\sigma_{0}-$ предел $\sigma$ при высокой температуре [25]. Температурная зависимость $\sigma(T)$ представлена на вставках к рис. 1. Полученные в результате аппроксимации параметры $\hbar \omega$ и $\sigma_{0}$ приведены в табл. 1. Согласно аппроксимации значения энергии $\hbar \omega$ в молибдатах составляют 50-90 meV. Эти значения 

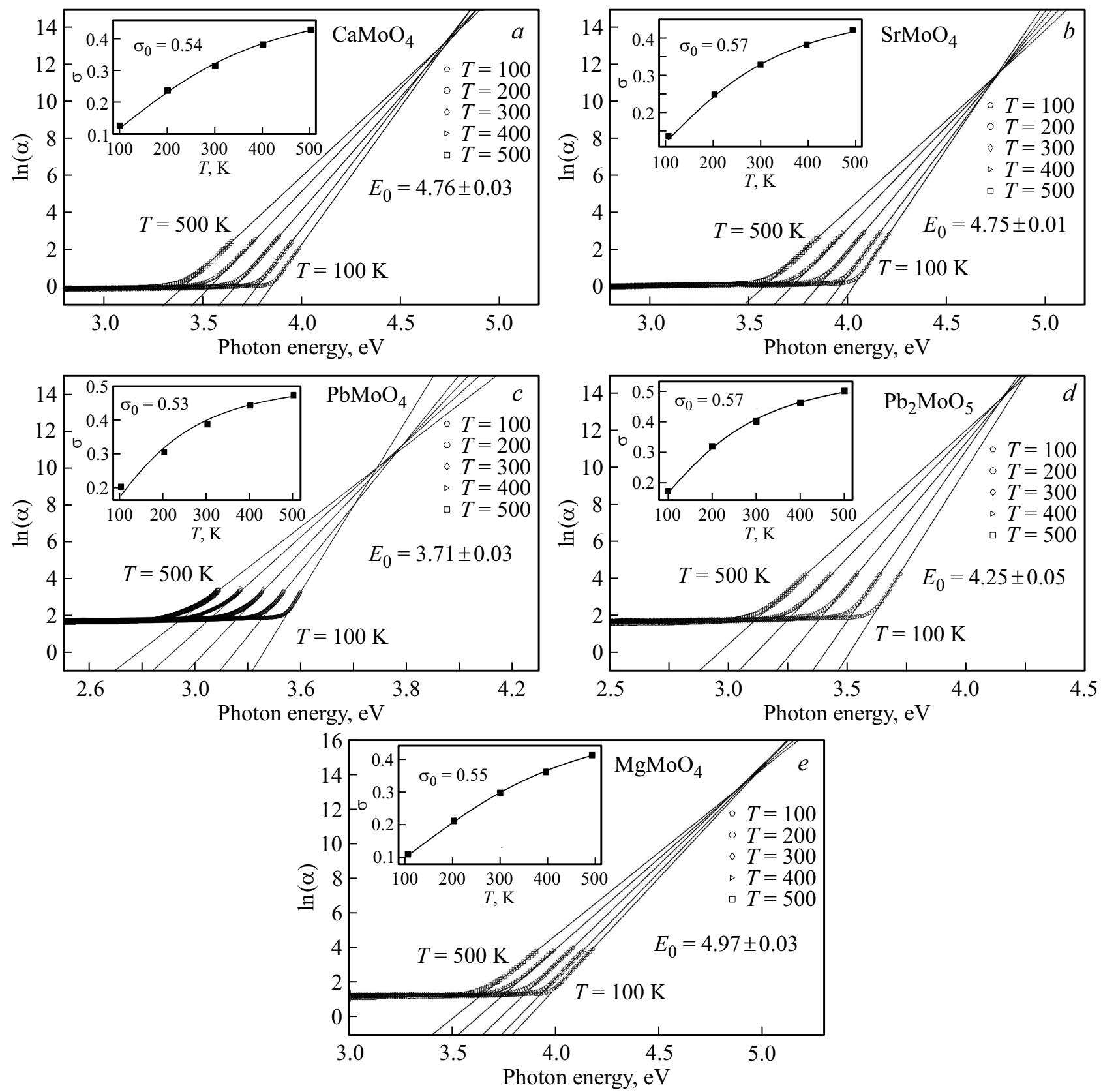

Рис. 1. Температурные зависимости коэффициента поглощения молибдатов. На вставках представлены зависимости $\sigma(T)$. $a-\mathrm{CaMoO}_{4}, b-\mathrm{SrMoO}_{4}, c-\mathrm{PbMoO}_{4}, d-\mathrm{Pb}_{2} \mathrm{MoO}_{5}, e-\mathrm{MgMoO}_{4}$. Результаты аппроксимации представлены линиями, экспериментальные данные - точками.

соответствуют диапазону характерных для молибдатов энергий оптических фононов [36-40].

Были также измерены спектры отражения в области края ФП молибдатов. Полученные результаты представлены на рис. 2. На рисунках приведены полученные выше значения $E_{0}$. Из представленных спектров следует, что пики отражения, соответствующие $E_{0}$, наблюдаются для свинецсодержащих молибдатов $\mathrm{PbMoO}_{4}$ и $\mathrm{Pb}_{2} \mathrm{MoO}_{5}$, тогда как для остальных молибдатов первые пики в спектрах отражения на $0.5-0.6 \mathrm{eV}$ смещены в высокоэнергетическую область.

\section{4. Обсуждение}

\section{1. Проявление экситонов в молибдатах}

Из представленных результатов следует, что температурная зависимость края ФП всех исследованных молибдатов описывается формулой (1). Ранее аппроксимация на основе экспериментальных спектров поглощения проводилась для кристаллов $\mathrm{PbMoO}_{4}[30,31]$ и $\mathrm{MgMoO}_{4}$ [15]. Значение $E_{0}$, полученное для молибдата свинца $3.71 \mathrm{eV}$, согласуется с результатами предыдущих 

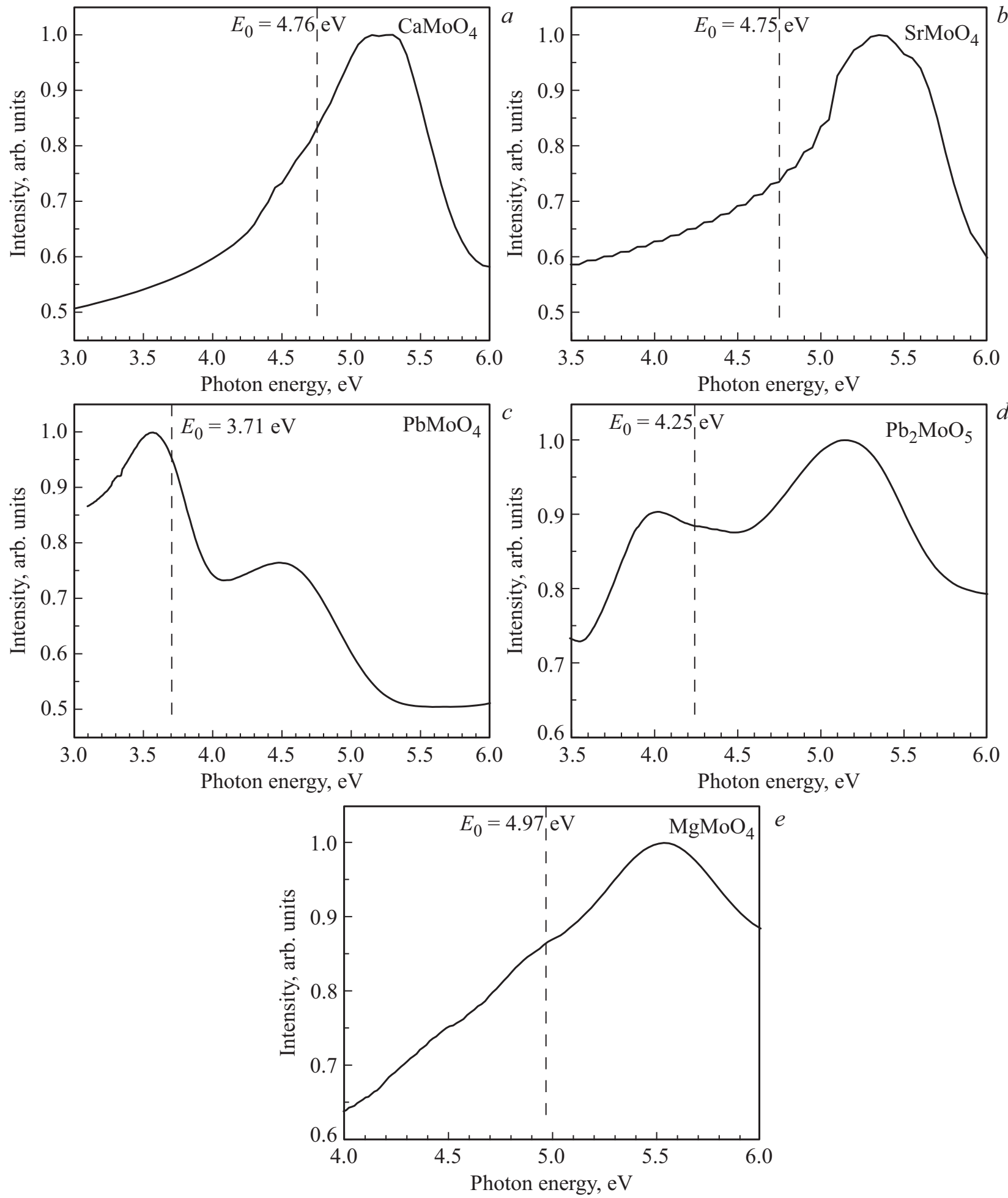

Pис. 2. Спектры отражения в области края фундаментального поглощения молибдатов, $T=300 \mathrm{~K}: a-\mathrm{CaMoO}_{4}, b-\mathrm{SrMoO}_{4}$, $c-\mathrm{PbMoO}_{4}, d-\mathrm{Pb}_{2} \mathrm{MoO}_{5}, e-\mathrm{MgMoO}_{4}$. Вертикальными линиями отмечены значения $E_{0}$, полученные из аппроксимации по формуле Урбаха.

исследований - $3.57 \mathrm{eV}$ [30] и $3.52 \mathrm{eV}$ [31], несмотря на наличие полос поглощения в области прозрачности исследованного нами образца $\mathrm{PbMoO}_{4}$. Значения $E_{0}$ для $\mathrm{MgMoO}_{4}-4.49 \mathrm{eV}(T=300 \mathrm{~K})$ и $4.57 \mathrm{eV}$
$(T=10 \mathrm{~K})$ заметно меньше, чем полученные в настоящей работе $-4.97 \mathrm{eV}$. Однако, в работе [15] значения $E_{0}$ для $\mathrm{MgMoO}_{4}$ были получены без определения точки пересечения аппроксимирующих кривых. 
Таблица 2. Литературные данные по значениям $E_{g}$ молибдатов, полученных разными методами

\begin{tabular}{|c|c|c|c|c|c|}
\hline \multirow{2}{*}{$\begin{array}{c}\text { Метод, } \\
\text { использованный } \\
\text { для оценки }\end{array}$} & \multicolumn{5}{|c|}{ Значение $E_{g}, \mathrm{eV}$} \\
\hline & $\mathrm{CaMoO}_{4}$ & $\mathrm{SrMoO}_{4}$ & $\mathrm{MgMoO}_{4}$ & $\mathrm{PbMoO}_{4}$ & $\mathrm{~Pb}_{2} \mathrm{MoO}_{5}$ \\
\hline $\begin{array}{l}\text { Построение Таука } \\
\text { для спектров оптического } \\
\text { поглощения или отражения }\end{array}$ & $\begin{array}{l}4.16^{*}[50] \\
4.5[51] \\
3.30^{*}[52] \\
3.725^{*}[53] \\
3.68[54] \\
5.07^{*}[55]\end{array}$ & $\begin{array}{l}3.51^{*}[52] \\
3.72^{*}[55] \\
3.98^{*}[57] \\
4.182^{*}[58] \\
3.7^{*}[59] \\
3.9^{*}[60] \\
3.92[61] \\
3.98^{*}[62] \\
4.16^{*}[63]\end{array}$ & $\begin{array}{l}5.15[65] \\
3.23[66]\end{array}$ & $\begin{array}{c}3.20^{*}[67] \\
3.14-3.19[68] \\
3.21-3.24^{*}[69] \\
3.12^{*}[70] \\
3.85[71] \\
4.03[71]\end{array}$ & \\
\hline $\begin{array}{l}\text { По данным расчетов } \\
\text { зонной структуры }\end{array}$ & $3.41[17]$ & $\begin{array}{l}3.687[63] \\
4.46[64]\end{array}$ & - & $\begin{array}{c}2.838[72] \\
2.59[17] \\
3.4[42]\end{array}$ & $\begin{array}{l}2.41[41] \\
2.64[42]\end{array}$ \\
\hline $\begin{array}{l}\text { По положению } \\
\text { первого пика отражения }\end{array}$ & $5.1[56]$ & $5.3[56]$ & $5.2[15]$ & - & - \\
\hline $\begin{array}{l}\text { Расчеты зонной структуры, } \\
\text { исправленные с учетом } \\
\text { спектров отражения [49] }\end{array}$ & 4.4 & 4.7 & - & 3.3 & - \\
\hline
\end{tabular}

Примечание. * — результаты были получены для нанопорошков.

Согласно [25] значение $E_{0}$ определяет положение низкоэнергетического экситонного пика. Пики отражения с максимумами, близкими к полученным значениям $E_{0}$, наблюдаются только у свинецсодержащих молибдатов $\mathrm{PbMoO}_{4}$ и $\mathrm{Pb}_{2} \mathrm{MoO}_{5}$. Отличительной особенностью $\mathrm{PbMoO}_{4}$ является участие электронных состояний катиона в формировании дна зоны проводимости и потолка валентной зоны [30]. Также, согласно расчетам зонной структуры $\mathrm{Pb}_{2} \mathrm{MoO}_{5}$, электронные состояния $6 s \mathrm{~Pb}$ принимают участие в формировании потолка валентной зоны, тогда как состояния $6 p \mathrm{~Pb}$ - в формировании дна зоны проводимости $[41,42]$. Это позволяет предположить, что пики отражения, соответствующие значениям $E_{0}$ для $\mathrm{PbMoO}_{4}$ и $\mathrm{Pb}_{2} \mathrm{MoO}_{5}$, представляют собой катионные экситоны, создающиеся при квазиатомарном переходе ${ }^{1} S_{0} 6 s^{2} \rightarrow{ }^{3} P_{1} 6 s 6 p$ на ионе свинца $\mathrm{Pb}^{2+}$. Катионные экситоны на $\mathrm{Pb}^{2+}$ также ранее наблюдались в спектрах отражения ряда других свинецсодержащих кристаллов, таких как $\mathrm{PbWO}_{4}, \mathrm{PbCO}_{3}, \mathrm{PbSO}_{4}, \mathrm{PbF}_{2}$, $\mathrm{PbCl}_{2}$ [43-46]. Отметим, что значения $E_{0}$ соответствуют не точному положению максимумов первых пиков отражения $\mathrm{PbMoO}_{4}$ и $\mathrm{Pb}_{2} \mathrm{MoO}_{5}$, а их высокоэнергетическим спадам. Однако значение $E_{0}$ должно соответствовать максимуму экситонного пика при $T \rightarrow 0$, с увеличением температуры экситонный пик смещается в область высоких температур, что объясняет наблюдаемое расхождение.

Для других молибдатов первый пик отражения существенно (на 0.5-0.6 eV) смещен в высокоэнергетическую область относительно $E_{0}$. Это связывается с тем, что экситонные пики не проявляются в спектрах отражения $\mathrm{CaMoO}_{4}, \mathrm{SrMoO}_{4}$ и $\mathrm{MgMoO}_{4}$, а первые пики отражения связаны с межзонными электронными переходами из валентной зоны в зону проводимости.

Согласно [25], значение параметра $\sigma_{0}$ обратно пропорционально силе экситон-фононной связи. Параметр $\sigma_{0}$ является критерием автолокализации экситонов: при $\sigma_{0}<1$ экситоны в кристалле автолокализуются, при $\sigma_{0}>1$ экситоны в кристалле существуют в свободном состоянии. Полученные значения $\sigma_{0}$ заметно меньше единицы, что указывает на сильную экситон-фононную связь в молибдатах. Свечение автолокализованных экситонов представляет собой широкую полосу люминесценции с большим стоксовым сдвигом. Известно, что собственное свечение молибдатов характеризуется широкой полосой и стоксовым сдвигом и связывается с излучательной релаксацией автолокализованных экситонов [14-17]. Таким образом, вывод о сильной экситонфононной связи, который можно сделать по результатам аппроксимации, согласуется с наблюдаемым свечением автолокализованных экситонов в молибдатах.

\section{2. Оценка ширины запрещенной зоны молибдатов}

Значение ширины запрещенной зоны кристалла $E_{g}$ соответствует началу электронных переходов из валентной зоны в зону проводимости. $E_{g}$ является фундаментальным параметром соединения, который, в частности, используется для оценки его сцинтилляционного выхода [47]. Значения $E_{0}$, полученные в настоящей работе, могут быть использованы для оценки $E_{g}$ молибдатов. Отметим, что значения $E_{0}$ будут незначительно отличаться 
от $E_{g}$ на величину энергии связи экситонов. Так, для молибдата цинка энергия связи экситонов была оценена как $60 \mathrm{meV}$ [48].

Такой метод позволяет получить достаточно точную оценку $E_{g}$ по сравнению с рядом других методов оценки, использовавшихся ранее. В табл. 2 приведены литературные данные по значениям $E_{g}$ молибдатов. Как видно из таблицы, полученные значения $E_{g}$ существенно отличаются друг от друга. Например, для молибдата кальция были получены значения от 3.41 до $5.1 \mathrm{eV}$. Такой разброс значений связан с особенностями использованных методов оценки. Для экспериментального определения $E_{g}$ в большинстве случаев использовалось так называемое построение Таука - зависимость $(\alpha h v)^{1 / r}$ от $h v$, где $\alpha$ - коэффициент поглощения, а $r-$ коэффициент, зависящий от особенностей зонной структуры кристалла [73]. Ширина запрещенной зоны определяется по пересечению экстраполяции линейного участка роста $(\alpha h v)^{1 / r}$ с осью абсцисс. Отметим, что метод Таука основан на упрощенном описании межзонного поглощения для случая полупроводников с параболическими ветвями зоны проводимости и валентной зоны и, строго говоря, не может применяться при наличии экситонных эффектов. Несмотря на это, его часто используют вне пределов применимости как „инженерный“ подход к оценке оптической ширины запрещенной зоны, то есть энергетического значения, при котором кристалл перестает быть прозрачным. Оптическая ширина запрещенной зоны зависит как от качества образцов, так и от параметров измерений (например, от толщины образца), что определяет наблюдаемый разброс полученных значений $E_{g}$. Значения $E_{g}$, полученные с использованием такого построения, не учитывают создание экситонов в области края ФП и дают значения меньшие, чем $E_{0}$.

В работе [56] оценка $E_{g}$ для $\mathrm{CaMoO}_{4}$ и $\mathrm{SrMoO}_{4}$ была проведена по положению максимума первого пика в спектре отражения в области ФП. Как следует из результатов настоящей работы, первые пики отражения в этих молибдатах связаны с межзонными электронными переходами. Однако значение $E_{g}$ соответствует началу межзонных переходов, в то время как при относительно слабых экситонных эффектах первый максимум поглощения (отражения) будет соответствовать переходам между первыми пиками плотностей электронных состояний в области потолка валентной зоны и дна зоны проводимости. Таким образом, положение первого пика отражения превышает реальное значение $E_{g}$.

Оценки, проведенные на основании теоретических расчетов зонной структуры, как правило, дают заниженные значения. Это связано с особенностями расчетов в рамках теории функционала плотности, которая обычно используется для этих целей [74].

В работе [49] $E_{g}$ определялась на основе совместного анализа результатов теоретических расчетов зонной структуры и экспериментальных спектров отражения. Корректировка рассчитанной $E_{g}$ проводилась путем смещения состояний зоны проводимости так, чтобы положе- ние пиков отражения расчетного спектра соответствовало экспериментальным. Сравнение значений $E_{0}$ с $E_{g}$, полученными в [49], показывает достаточно хорошее согласие для кристалла $\mathrm{SrMoO}_{4}(4.75$ и $4.7 \mathrm{eV})$, тогда как для $\mathrm{PbMoO}_{4}(3.71$ и $3.3 \mathrm{eV})$ и $\mathrm{CaMoO}_{4}(4.76$ и $4.4 \mathrm{eV})$ значение $E_{0}$ заметно превышает рассчитанные $E_{g}$. Мы полагаем, что значение $E_{g}$ для $\mathrm{PbMoO}_{4}$, полученное в [49], было недооценено. В этой работе первый пик отражения при $3.6 \mathrm{eV}$ был принят соответствующим межзонным электронным переходам. Однако результаты, представленные в настоящей работе, свидетельствуют в пользу его экситонной природы. Определение причины отличия значений для $\mathrm{CaMoO}_{4}$ требует проведения дополнительных исследований.

\section{5. Заключение}

Показано, что температурная зависимость края фундаментального поглощения молибдатов может быть аппроксимирована с использованием формулы Урбаха. Были определены значения энергии создания экситонов $E_{0}$ и параметра $\sigma_{0}$. Для всех молибдатов $\sigma_{0}<1$, что свидетельствует о сильной экситон-фононной связи и является критерием автолокализации экситонов. На основе анализа литературных данных сделан вывод, что значение $E_{0}$ может быть использовано для оценки ширины запрещенной зоны $E_{g}$ молибдатов. Среди молибдатов выделяются кристаллы, содержащие свинец - $\mathrm{PbMoO}_{4}$ и $\mathrm{Pb}_{2} \mathrm{MoO}_{5}$. Эти молибдаты характеризуются наименьшими среди исследованных молибдатов значениями $E_{g}$, а также наличием в спектрах отражения пиков, близких по энергии к $E_{0}$. Эти пики связываются с катионными экситонами, и их появление обусловлено участием энергетических уровней $\mathrm{Pb}^{2+}$ в формировании дна зоны проводимости и потолка валентной зоны.

\section{Благодарности}

Авторы благодарны О.А. Бузанову за предоставление кристалла $\mathrm{CaMoO}_{4}$, Л.И. Ивлевой за предоставление кристаллов $\mathrm{MgMoO}_{4}, \mathrm{SrMoO}_{4}$, Б.И. Заднепровскому за предоставление кристалла $\mathrm{PbMoO}_{4}$ и В.Н. Шлегелю за предоставление кристалла $\mathrm{Pb}_{2} \mathrm{MoO}_{5}$.

\section{Конфликт интересов}

Авторы заявляют, что у них нет конфликта интересов.

\section{Список литературы}

[1] P. Belli, A. Incicchitti, F. Cappella. Int. J. Mod. Phys. A 29, 1443011 (2014).

[2] E. Armengaud, C. Augier, A.S. Barabash, J.W. Beeman, T.B. Bekker, F. Bellini, A. Benoit, L. Bergé, T. Bergmann, J. Billard, R.S. Boiko, A. Broniatowski, V. Brudanin, P. Camus, S. Capelli, L. Cardani, N. Casali, A. Cazes, M. Chapellier, F. Charlieux, D.M. Chernyak, M. de Combarieu, N. Coron, 
F.A. Danevich, I. Dafinei, M. De Jesus, L. Devoyon, S. DiDomizio, L. Dumoulin, K. Eitel, C. Enss, F. Ferroni, A. Fleischmann, N. Foerster, J. Gascon, L. Gastaldo, L. Gironi, A. Giuliani, V.D. Grigorieva, M. Gros, L. Hehn, S. Hervé, V. Humbert, N.V. Ivannikova, I.M. Ivanov, Y. Jin, A. Juillard, M. Kleifges, V.V. Kobychev, S.I. Konovalov, F. Koskas, V. Kozlov, H. Kraus, V.A. Kudryavtsev, M. Laubenstein, H. Le Sueur, M. Loidl, P. Magnier, E.P. Makarov, M. Mancuso, P. de Marcillac, S. Marnieros, C. MarracheKikuchi, S. Nagorny, X-F. Navick, M.O. Nikolaichuk, C. Nones, V. Novati, E. Olivieri, L. Pagnanini, P. Pari, L. Pattavina, M. Pavan, B. Paul, Y. Penichot, G. Pessina, G. Piperno, S. Pirro, O. Plantevin, D.V. Poda, E. Queguiner, T. Redon, M. Rodrigues, S. Rozov, C. Rusconi, V. Sanglard, K. Schäffner, S. Scorza, V.N. Shlegel, B. Siebenborn, O. Strazzer, D. Tcherniakhovski, C. Tomei, V.I. Tretyak, V.I. Umatov, L. Vagneron, Ya.V. Vasiliev, M. Velázquez, M. Vignati, M. Weber, E. Yakushev, A.S. Zolotarova. Eur. Phys. J. C 77, 785 (2017).

[3] S. Belogurov, V. Kornoukhov, A. Annenkov, A. Borisevich, A. Fedorov, M. Korzhik, V. Ligoun, O. Missevitch, S.K. Kim, S.C. Kim, S.Y. Kim, J.W. Kwak, H.S. Lee, J. Lee, S.S. Myung, M.J. Lee, Y.D. Kim, J.Y. Lee, J.I. Lee, H.J. Kim, Y.J. Kwon, M.J. Hwang, J.J. Zhu. IEEE T Nukl. Sci. 52, 1131 (2005).

[4] F.A. Danevich. IEEE T Nukl. Sci. 59, 2207 (2012).

[5] M. Tenconi. Phys. Proc. 61, 782 (2015).

[6] J.D. Vergados, H. Ejiri, F Simkovic. Rep. Prog. Phys. 75, 106301 (2012).

[7] E. Fiorini. Nucl. Phys. B 110, 233 (2002).

[8] D.A. Spassky, V.V. Alenkov, O.A. Buzanov, V.N. Kornoukhov. Springer Proc. Phys. 200, 242 (2017).

[9] H. Kim, I.R. Pandey, A. Khan, J. Son, M.H. Lee, Y. Kim. Cryst. Res. Technol. 54, 1900079 (2019).

[10] V.B. Mikhailik, H. Kraus. Phys. Status Solidi B 7, 1583 (2010).

[11] H.L. Kim, J.A. Jeon, I. Kim, S.R. Kim, H.J. Kim, Y.H. Kim, D.H. Kwon, M.K. Lee, J.H. So. Nucl. Instrum. Meth. A 954, 162107 (2019).

[12] T.B. Bekker, N. Coron, F.A. Danevich, V.Ya. Degoda, A. Giuliani, V.D. Grigorieva, N.V. Ivannikova, M. Mancuso, P. de Marcillac, I.M. Moroz, C. Nones, E. Olivier, G. Pessina, D.V. Poda, V.N. Shlegel, V.I. Tretyak, M. Velazquez. Astropart. Phys. 72, 38 (2016).

[13] V.D. Grigorieva, V.N. Shlegel, N.V. Ivannikova, T.B. Bekker, A.P. Yelisseyev, A.B. Kuznetsov. J. Cryst. Growth 507, 31 (2019).

[14] Е.Г. Реут. Изв. АН СССР. Сер. физ. 49, 2032 (1985).

[15] Д.А. Спасский, В.Н. Колобанов, В.В. Михайлин, Л.Ю. Березовская, Л.И. Ивлева, И.С. Воронина. Оптика и спектроскопия 106, 622 (2009).

[16] V.B. Mikhailik, H. Kraus, M. Itoh, D. Iri, M. Uchida. J. Phys.: Condens. Matter 17, 7209 (2005).

[17] Y. Zhang, N.A.W. Holzwarth, R.T. Williams. Phys. Rev. B 57, 12738 (1998).

[18] D.A. Spassky, V. Nagirnyi, V.V. Mikhailin, A.E. Savon, A.N. Belsky, V.V. Laguta, M. Buryi, E.N. Galashov, V.N. Shlegel, I.S. Voronina, B.I. Zadneprovski. Opt. Mater. 35, 2465 (2013).

[19] V.B. Mikhailik, Yu. Elyashevskyi, H. Kraus, H.J. Kim, V. Kapustianyk, M. Panasyuk. Nucl. Instrum. Meth. A 792, 1 (2015).
[20] V.B. Mikhailik, H. Kraus. Phys. Status Solidi B 247, 1583 (2010).

[21] E. Zych, C. Brecher, J. Glodo. J. Phys.: Condens. Matter 12, 1947 (2000).

[22] S. Gridin, A. Belsky, C. Dujardin, A. Gektin, N. Shiran, A. Vasil'ev. Phys. Chem. C 119, 20578 (2015).

[23] C. Sailer, B. Lubsandorzhiev, C. Strandhagen, J. Jochum. Eur. Phys. J. 72, 2061 (2012).

[24] L. Swiderski, M. Moszyński, W. Czarnacki, K. Brylew, M. Grodzicka-Kobylka, Z. Mianowska, T. Sworobowicz, A. Syntfeld-Kazuch, T. Szczesniak, W. Klamra, R.T. Williams, S. Gridin, X. Lu, M.R. Mayhugh, A. Gektin, S. Vasyukov, C. Piemonte, F. Acerbi, A. Ferri, A. Gola, T. Zawistowski. Nucl. Instrum. Meth. 916, 32 (2019).

[25] K.S. Song, R.T. Williams. Self-Trapped Excitons. 2nd ed. Springer Ser. Solid-State Sci. V. 105. Springer-Verlag, Berlin (1996). $404 \mathrm{p}$.

[26] I. Studenyak, M. Kranjčec, M. Kurik. Int. J. Opt. Appl. 4, 3, 76 (2014).

[27] F. Urbach. Phys. Rev. 92, 1324 (1953).

[28] Y. Toyozawa. Tech. Rep. ISSP A 1, 119, 1 (1964).

[29] M. Schreiber, Yu. Toyozawa. J. Phys. Soc. Jpn, 51, 1544 (1982).

[30] M. Fujita, M. Itoh. Phys. Status Solidi B 247, 2240 (2010).

[31] W. Van Loo. Phys. Status Solidi A 27, 565 (1975).

[32] E. Gurmen, E. Daniels, J.S King. J. Chem. Phys. 55, 1093 (1971).

[33] В.В. Бабакин, Р.Ф. Клевцова, Л.А. Гапоненко. Кристаллография 27, 38 (1982).

[34] S. Miyazawa, H. Iwasaki. J. Cryst. Growth 8, 359 (1971).

[35] И.В. Митин, В.С. Русаков. Анализ и обработка экспериментальных данных. Изд-во НЭВЦ ФИПТ, М. (1998). 48 с.

[36] K. Kesavasamy, N. Krishnamurthy. Can. J. Phys. 60, 1447 (1982).

[37] T. Sinagawa, J. Suda, T. Sato, H. Saito. J. Phys. Soc. Jpn. 69, 464 (2000).

[38] T. Thongtem, A. Phuruangrat, S. Thongtem. Mater. Lett. 62, 454 (2008).

[39] Y. Liang, P. Liu, H. B. Li, G. W. Yang. Cryst. Growth Des. 12, 4487 (2012).

[40] P.J. Miller. Spectrochim. Acta 27A, 957 (1971).

[41] O.Y. Khyzhun, V.L. Bekenev, V.V. Atuchin, L.D. Pokrovsky, V.N. Shlegel, N.V. Ivannikova. Mater. Des. 105, 315 (2016).

[42] S. Nedilko, V. Chornii, Yu. Hizhnyi, M. Trubitsyn, I. Volñyanskaya. Opt. Mater. 36, 1754 (2014).

[43] M. Fujita, H. Nakagawa, K. Fukui, H. Matsumoto, T. Miyanaga, M. Watanabe. J. Phys. Soc. Jpn. 60, 4393 (1991).

[44] M. Fujita, M. Itoh, H. Nakagawa, M. Kitaura, D. Alov. J. Phys. Soc. Jpn. 67, 3320 (1998).

[45] I.A. Kamenskikh, M. Kirm, V.N. Kolobanov, V.V. Mikhailin, P.A. Orekhanov, I.N. Shpinkov, D.A. Spassky, A. N. Vasil'ev, B.I. Zadneprovsky, G. Zimmerer. IEEE T NUCL SCI 48, 2324 (2001).

[46] I.A. Kamenskikh, M. Kirm, V.N. Kolobanov, V.V. Mikhailin, P.A. Orekhanov, I.N. Shpinkov, D.A. Spassky A.N. Vasil'ev, G. Zimmerer. Radiat. Eff. Defect. S 154, 307 (2001).

[47] M. Nikl. Meas. Sci. Technol. 17, R37 (2006).

[48] D.A. Spassky, A.N. Vasil'ev, I.A. Kamenskikh, V.V. Mikhailin, A.E. Savon, Yu.A. Hizhnyi, S.G. Nedilko, P.A. Lykov. J. Phys.: Condens. Matter 23, 365501 (2011).

[49] D.A. Spassky, N.S. Kozlova, V. Nagirnyi, A.E. Savon, Yu.A. Hizhnyi, S.G. Nedilko. J. Lumin. 186, 229 (2017). 
[50] В.В. Баковец, И.В. Юшина, О.В. Антонова, Е.С. Золотова. Оптика и спектроскопия 123, 372 (2017).

[51] V. Panchal, N. Garg, H.K. Poswal, D. Errandonea, P. Rodríguez-Hernández, A. Muñoz, E. Cavalli. Phys. Rev. Mater. 1, 043605 (2017).

[52] S.D. Ramarao, S. Roopas Kiran, V.R.K. Murthy. Mater. Res. Bull. 56, 71 (2014).

[53] S. Vidya, S. Solomon, J.K. Thomas. Phys. Status Solidi A 209, 1067 (2012).

[54] E. Sinha, P. Yadav. Ferroelectrics 517, 193 (2017).

[55] T. Thongtem, S. Kungwankunakorn, B. Kuntalue, A. Phuruangrat, S. Thongtem. J. Alloy. Compd. 506, 475 (2010).

[56] D. Spassky, S. Ivanov, I. Kitaeva, V. Kolobanov, V. Mikhailin, L. Ivleva, I. Voronina. Phys. Status Solidi C 2, 65 (2005).

[57] J. Luo, X. Bai, Q. Li, X. Yu, C. Li, Z. Wang, W. Wu, Y. Liang, Z. Zhao, H. Liu. Nano Energy 66, 104187 (2019).

[58] M.M.S. Silva, M.S. Sena, A.L. Lopes-Moriyama, C.P. Souza, A.G. Santos. Ceram. Int. 44, 16606 (2018).

[59] S. Vidya, A. John, S. Solomon, J.K. Thomas. Adv. Mater. Res. 1, 191 (2012).

[60] Z.F. Yao, G.H. Zheng, Z.X. Dai, L.Y. Zhang. Appl. Organomet. Chem. 32, e4412 (2018).

[61] S. Wannapop, T. Thongtem, S. Thongtem. J. Nanomater. 2013, 474576 (2013).

[62] J.C. Sczancoski, L.S. Cavalcante, M.R. Joya, J.A. Varela, P.S. Pizani, E. Longo. Chem. Eng. J. 140, 632 (2008).

[63] L. Li, Y. Pan, W. Chang, Z. Feng, P. Chen, C. Li, Z. Zeng, X. Zhou. Mater. Res. Bull. 93, 144 (2017).

[64] J. Yin, Q. Zhang, T. Liu, X. Guo, M. Song, X. Wang, H. Zhang. Curr. Appl. Phys. 9, 1237 (2009).

[65] S. Wannapop, T. Thongtem, S. Thongtem. J. Phys. Chem. Solids 74, 677 (2013).

[66] C.S. Xavier, A.P. de Moura, E.Longo, J.A. Varela, M.A. Zaghete. Adv. Mat. Res. 975, 243 (2014).

[67] S. Vidya, J.K. Thomas. IOP. Conf. Ser-Mat Sci. 73, 012120 (2015).

[68] J.C. Sczancoski, M.D.R. Bomio, L.S. Cavalcante, M.R. Joya, P.S. Pizani, J.A. Varela, E. Longo, M. Siu Li, J.A. Andres. J. Phys. Chem. C 113, 5812 (2009).

[69] G.M. Gurgel, L.X. Lovisa, L.M. Pereira, F.V. Motta, M.S. Li, E. Longo, C.A. Paskocimas, M.R.D. Bomio. J. Alloy Compd. 700, 130 (2017).

[70] T.K. Thirumalaisamy, R.J. Saravanan. J. Mater Sci.: Mater Electron 22, 1637 (2011).

[71] R. Jia, Y. Zhang. Chin. Opt. Lett. 8, 1152 (2010).

[72] Q.J. Liu, Z.T. Liu, L.P. Feng, H. Tian. ISRN Condens. Matter Phys. 2011, 290741 (2011).

[73] D.L. Wood, J. Tauc. Phys. Rev. B 5, 3144 (1972).

[74] T. Ziegler. Chem. Rev. 91, 651 (1991).

Редактор Т.Н. Василевская 